\title{
Teknokultura
}

ISSNe: $1549-2230$

http://dx.doi.org/10.5209/TEKN.57194

\section{Transparentar el conocimiento urbano para el apoyo a la decisión mediante inteligencia artificial: comprendiendo la percepción infantil de los entornos escolares de Granada ${ }^{1}$}

\author{
Francisco Javier Abarca-Álvarez²; Rubén Mora-Esteban³; Francisco Sergio Campos-Sánchez ${ }^{4}$
}

Recibido: 15 de septiembre de 2017 / Revisado: 24 de noviembre de 2017 / Renviado para revisión: 6 de diciembre de 2017 / Aceptado: 26 de diciembre de 2017. Open peer reviews

Resumen. Las recientes trasformaciones socio-económicas están incentivando sociedades que aspiran a roles más activos en la toma de decisiones, afectando a las organizaciones sociales, pero también a la gestión del conocimiento y la educación. Para que ocurra este empoderamiento es necesario un mejor y más equitativo acceso a la información, más transparente, comprensible e interpretable. El trabajo que se presenta continúa una Investigación-Participación realizada con universitarios y otros ciudadanos que permitió consensuar atributos e indicadores de la calidad de vida y sostenibilidad urbana. La investigación se enfoca ahora en el objetivo de aportar conocimiento útil para el empoderamiento ciudadano en la toma de decisiones. Como caso de estudio se seleccionan los entornos urbanos escolares de la ciudad de Granada. La información se obtiene mediante una metodología cuantitativa con formularios a niños sobre sus dimensiones: familiar, residencial, relación con el medio, y perceptiva del entorno urbano colegial. Usando técnicas de Inteligencia Artificial sobre las tres primeras dimensiones se obtienen perfiles de niños atendiendo a tales realidades. Los perfiles se contrastan con la dimensión perceptiva que los niños tienen del ámbito próximo a su colegio, representándose finalmente de manera comprensible para la interpretación directa por la ciudadanía. Se concluye que la percepción infantil de la ciudad está influenciada por el contexto familiar y especialmente por el tipo de desplazamientos que realiza, siendo generalmente más positiva cuando éstos son peatonales. Ello enraíza con la relevancia de las ideas de la Ciudad de los Niños, más peatonal y humana, legitimando líneas de acción crítica ciudadana.

Palabras clave: apoyo a la decisión; ciudad educadora; empoderamiento ciudadano; investigaciónacción; sostenibilidad urbana.

[en] Transparent knowledge for decision support through artificial intelligence: Understanding the perception of children in Granada's school environments

Abstract. Recent socio-economic transformations are encouraging societies that aspire to more active decision-making roles, affecting social organizations, but also knowledge management

1 Este proyecto ha sido parcialmente financiado por el proyecto V1-2015 de la III Convocatoria de Microproyectos de $\mathrm{I}+\mathrm{D}+\mathrm{i}$ del Campus de Excelencia Internacional BIOTIC Granada, Universidad de Granada, titulado "Laboratorio urbano de Mapas auto-organizados (UrbanLabSOM). Influencia de la forma del patrimonio urbanístico de Granada en la sostenibilidad y eco-eficiencia de las barriadas y en su percepción social, mediante el uso de redes neuronales artificiales y mapas auto-organizados (SOM).

2 Universidad de Granada (España)

E-mail: fcoabarca@ugr.es

3 Universidad Politécnica de Madrid (España)

E-mail: rumorest@gmail.com

4 Universidad de Granada (España)

E-mail: scampos@ugr.es 
and education. For this empowerment to occur, better and more equitable access to information, more transparent, understandable and interpretable, is needed. This work continues a Participation Research carried out with university students and other citizens. This research allowed consensus to be reached on attributes and indicators of the quality of life and urban sustainability. Research is now focused on trying to provide useful knowledge for citizen empowerment in decision-making. The urban school environments of the city of Granada are selected as a case study. The information is obtained by means of a quantitative methodology with forms to children on their family and residential dimensions, relationship with the environment and perceptive of the urban school environment. Using Artificial Intelligence techniques on the first three dimensions, profiles of children are obtained according to these realities. The profiles are contrasted with the perceptive dimension that children have of the environment close to their school, and finally represented in a comprehensible way for direct interpretation by citizens. It is concluded that the child's perception of the city is influenced by the family context and especially by the type of displacements it makes, being generally more positive when these are pedestrian. This is rooted in the relevance of 'Children's City' ideas, which are more pedestrian and humane, legitimizing lines of critical citizen action.

Keywords: action-research; citizen empowerment; decision Support System; educating city; urban sustainability.

Sumario: 1. Introducción. 2. Material y método. 3. Análisis y resultados. 4. Discusión y conclusiones. 5. Referencias.

Cómo citar: Abarca-Álvarez, F.J.; Mora Esteban, R.; Campos Sánchez, F.S. “Transparentar el conocimiento urbano para el apoyo a la decisión mediante inteligencia artificial: Comprendiendo la percepción infantil de los entornos escolares de Granada": Teknokultura, vol. 15 (1) 2018, pp. 89-104.

\section{Introducción}

Es reconocido que la sociedad occidental actual se encuentra en una difícil coyuntura que anuncia nuevos paradigmas, siendo urgentes reflexiones renovadas sobre los procedimientos que la regulan. En este sentido se viene esbozando el germen de una ciudadanía mejor comunicada, más crítica y que demanda una mayor y más activa participación en los asuntos y las decisiones que le concierne.

Nos encontramos ante el conjunto de diversas crisis, todas ellas de claro origen antropogénico (Riechmann, 2011), que nos afectan sustancialmente: a) la económica, derivada de la globalización financiera tardocapitalista; b) la medioambiental, relacionada con el cambio climático y la biodiversidad; y c) la crisis social de la desigualdad, responsable de una "modernidad líquida" donde los vínculos entre las elecciones individuales y los proyectos colectivos se han disuelto (Bauman, 2000). Esta nueva situación ha desgarrado muchas de las certezas y certidumbres que construyeron la Europa Occidental posterior a la Segunda Guerra Mundial, donde el progreso económico se combinó con la mejora de las condiciones de vida, anticipando la sociedad del bienestar.

Entre las causas y los efectos de esta triple crisis encontramos como factor determinante las transformaciones urbanas y los factores espaciales al entender que la ciudad y el territorio están en el centro del diálogo entre la sociedad y el medio físico (Nel-Lo, 2015). Por esta razón la ciudad, que ha sido tradicionalmente considerada como un espacio propicio para la vida en común y la redistribución social, tiende hoy a fragmentarse desigualmente y a negar a muchos de sus habitantes la promesa 
del bienestar social y la posibilidad de desarrollar un proyecto de vida autónomo, o dicho en palabras de Sassen (2013), está perdiendo "su urbanidad".

Como resultado de este contexto de crisis socio-ecosistémica, se ha generalizado la decepción y la desconfianza hacia la gobernanza actual (Bray y McLaughlin, 2005), poniendo de manifiesto una creciente demanda de la sociedad en la participación de las decisiones de política pública (Ríos et al., 2008). Se cuestionan las maneras de actuación políticas, sociales, culturales y profesionales, ante la emergencia del creciente reclamo de derechos más colectivos y de prácticas más colaborativas.

Al mismo tiempo, asistimos a un desarrollo de las tecnologías de la información que avanzan inexorablemente cambiando los hábitos de vida, el empleo del tiempo y las relaciones personales (Castells, 1995), pero que a la vez constituyen el soporte comunicativo necesario para que todas estas reivindicaciones cobren forma en el ámbito cotidiano de manera organizada. En todo ello, la ciudad actúa como el soporte espacial físico necesario para el desarrollo de esta "revolución urbana" con la que la sociedad reclama sus derechos (Garnier, 2006; Harvey, 2013; Lefebvre, 1968).

Esto quedó patente en España con el movimiento ciudadano 15M, que establecía similitudes con el movimiento internacional surgido tras la Primavera Árabe, ambos en 2011. A partir de la explosión de un clima social ampliamente extendido, surge como una forma organizativa sin estructura, ocupando espacios públicos como las "espumas" de Sloterdijk (2006), y como expresión de la emergencia de nuevos escenarios y maneras de proceder. Desde su heterogeneidad, este movimiento supuso un cambio en la manera de entender las formas de participación política y otras maneras de politización de la experiencia colectiva, reclamando en el hacer público: transparencia, apertura, horizontalidad, democracia directa y prácticas de autonomía; nuevos procedimientos para el gobierno de sí mismo (Hessel, 2011).

Algunos autores interpretan estos movimientos como modelos idealizados de democracia directa asociada con la antigua Atenas (Crick, 2002); o modelos situados entre éste y la democracia representativa imperante. En ellos puede variar el grado de participación ciudadana, que conlleva una mayor legitimación y aceptación de las decisiones públicas, mayor transparencia y eficiencia en el gasto público, lo que se simultanea con una mayor satisfacción de los ciudadanos, enfatizando el aprendizaje y animando a considerar las preferencias e intereses colectivos, justificando y transformado las preferencias individuales (Baierle y Cayford, 2001). Cuanto mayor sea el grado de participación mayor será el empoderamiento ciudadano. Esto es mayor conciencia y control sobre los procesos que inciden o pueden incidir en su calidad de vida inmediata.

Otros autores, como Rifkin (2014), anuncian el nacimiento de un cambio de paradigma al afirmar la emergencia de un sistema económico nuevo en la escena mundial: el "procomún colaborativo". Paradigma de la incorporación de las ciencias sociales, humanas y biológicas a la construcción de los espacios habitables a través de un pensamiento más ecosistémico. Como anuncia Harvey (2013, p. 20): "La cuestión sobre qué tipo de ciudades deseamos no se puede separar de la cuestión sobre qué tipo de personas queremos ser, qué clase de relaciones sociales buscamos, qué relación con la naturaleza apreciamos, qué estilo de vida deseamos o a cuáles valores estéticos nos aferramos". Ciudad, educación y comunicación deben tratarse de un modo simultáneo e indisociable para afrontar los retos de esta nueva época.

Desde hace décadas se viene experimentando con las cualidades colaborativas y generadoras de comunidades autocríticas del tipo investigación-acción (Kemmis y 
McTaggart, 1988), mediante un proceso espiral, introspectivo y sistémico de aprendizaje. Según Thiollent (1981), las experiencias de investigación-acción se desarrollan a partir de las de Paulo Freire, en las que la educación es concebida desde una perspectiva concienciadora. Para Freire, "la concienciación es un proceso humano de auto-conocimiento, de reflexión sobre el mundo y de reconocimiento como protagonista de su propio destino" (Freire, 1970:5). Se conforma así una metodología para la intervención, desarrollo y cambio en grupos y comunidades, muy útil para la revisión de la división social del saber y del poder entre las partes implicadas (Goyette y Lessard-Hébert, 1988), y para la consolidación de unos nuevos roles en los que el ciudadano se implica más en las decisiones que le afectan. Además, la decisión legítima y deliberada, conjuntamente con la presunción del acceso libre y abierto y la transformación de las preferencias y objetivos mediante el debate, constituyen los pilares del discurso democrático (Habermas, 1994; Dryzek, 1990).

En este ámbito también son importantes las líneas de trabajo orientadas a contextos complejos en los que la información tiende a ser un bien valioso y no siempre bien manejado. Entre ellas encontramos los Sistemas de Apoyo a la Decisión (Decision Support System - DSS - ) que son medios eficaces para la integración de problemas complejos y el apoyo a la toma de decisiones donde es fundamental la correcta definición y el porqué de lo que se desarrolla (Keen, 1987). No son necesariamente sistemas informáticos, pero la actual proliferación masiva de información a manejar los aproxima a este ámbito. Existen cinco tipos de DSS según su orientación (Power et al., 2002): comunicación, datos, documentos, modelos y conocimiento, pero nuestro caso de estudio se enfoca fundamentalmente en el último de ellos, entendiendo el conocimiento como el germen del empoderamiento.

Resulta relevante la aportación de Carlsson y El Sawy (2008) sobre las "tensiones" que las tecnologías de la información deben gestionar en los procesos de decisión: velocidad frente a necesidad de proceso, capacidad de empoderamiento multinivel de usuarios, decisión frente a seguridad, o problemática entre atomización o centralización de la información. Los DSS se han usado en problemáticas urbanas junto con el análisis multicriterio multicapa (Feng y Xu, 1997); o de manera más reciente, integrados con Sistemas de Información Geográfica (GIS) para el apoyo a la decisión sobre políticas de transporte (Arampatzis, Kiranoudis, Scaloubacas y Assimacopoulos, 2004).

Alrededor de los DSS se han concentrado numerosos desarrollos tecnológicos, como por ejemplo los Sistemas de Conocimiento a partir de Bases de Datos (Knowledge Discovery in Databases - KDD_-). Originariamente los KDD no fueron pensados como disciplina propia, sino más bien como metodología de inteligencia para decisiones a nivel productivo y medioambiental (Cao et al, 2009), aunque con el tiempo se ha conformado como toda una ciencia (Data Science).

Encontramos múltiples técnicas procedentes de las ciencias de datos como constructoras de DSS y motores de KDD. Destacan algunas derivadas del campo de la Inteligencia Artificial (1956). Una de las técnicas que está experimentando mayor desarrollo y aplicación en los últimos años es la de Redes Neuronales Artificiales (Artificial Neural Networks - ANN-). Las ANNs están demostrando ser muy eficaces en la comprensión y resolución de problemas complejos en los que se necesita obtener información relevante a partir de múltiples variables, a veces heterogéneas y en ocasiones con valores perdidos o defectuosos. En nuestro caso de estudio se hace uso de un tipo concreto de ellas: los Mapas Auto-organizados (Self Organizing-Maps 
- SOM-) o Mapas de Kohonen (1989). Se caracterizan por presentar un aprendizaje no supervisado para el entrenamiento de la red.

Numerosas investigaciones han usado con éxito los SOM, como por ejemplo: a) auto-organización de datos (White y Engelen, 1993; Irwin y Geoghegan, 2001); b) geocomputación y optimización (Openshaw y Abrahart, 2000); c) integración con GIS (Wu, 1998); d) análisis de grupos sociales y barriadas (Spielman y Thill, 2008); e) análisis de sistemas urbanos (Kropp, 1998); f) la identificación y caracterización del urban sprawl (Diappi et al, 2004); g) patrones de transformación urbana de ciudades europeas (Hagen, Zanker y Timmermans, 2009); h) caracterización de tejidos urbanos (Abarca-Alvarez y Fernández-Avidad, 2010; Abarca-Alvarez, 2011); i) cartografías semánticas de barrios ejemplares europeos (Abarca-Alvarez y OsunaPérez, 2013); j) apoyo a la decisión en herramientas de gobierno electrónico (de Almeida-Gago, 2013); o k) la caracterización de las inmigraciones turísticas a partir de información censal (Abarca-Álvarez, Campos-Sánchez y Osuna-Pérez).

En torno a estas consideraciones, el objetivo principal del presente trabajo consiste en la obtención de conocimiento útil para la toma de decisiones y el empoderamiento ciudadano, usando para ello información sobre la percepción infantil de los entornos urbanos escolares e incorporando variables de las dimensiones familiares, domésticas y urbanas. Se pretende de este modo aportar conocimiento generado desde abajo hacia arriba, valioso para el ciudadano y su empoderamiento en la toma de decisiones de los asuntos que le afectan en su cotidianeidad.

\section{Material y método}

La investigación que se presenta en este artículo forma parte de una investigación de mayor tamaño, caracterizada por presentar una primera etapa consistente en una Investigación-Participación. Ésta se basó en la incorporación de una institución docente universitaria (estudiantes de Urbanismo de la Escuela Técnica Superior de Arquitectura de la Universidad de Granada) a una problemática real, interaccionando con múltiples agentes implicados en los entornos escolares de la ciudad y logrando el consenso de los atributos e indicadores de la calidad de vida y sostenibilidad urbana. La segunda etapa del trabajo, que es la que se presenta en este documento, se orienta hacia metodologías de Investigación-Acción: se integran acciones formativas y docentes del colectivo mencionado con escolares de Primaria de colegios públicos de Granada, intentando generar contextos de concienciación y transformación. Se distingue aquí entre Investigación-Participación e Investigación-Acción, aunque en el contexto internacional hay cierta discrepancia sobre la conveniencia de tal diferenciación (Oliveira de Vasconcelos y Waldenez de Oliveira, 2010). En este tipo de investigación hay que evitar los riesgos anunciados por Thiollent (1981) mediante una evaluación y revisión crítica sistemática en las distintas fases, no quedando sometida la investigación a exigencias de ninguna organización.

El objetivo principal de la investigación, tal y como se ha apuntado, es aportar conocimiento útil para la toma de decisiones y el empoderamiento ciudadano, basado éste en la percepción infantil sobre entornos urbanos escolares. Este conocimiento está orientado a posibilitar el empoderamiento ciudadano en lo concerniente a asuntos que le afectan en su día a día y sobre los cuales pueden tomar decisiones en las que habitualmente no estaban involucrados. Estas decisiones mediante la acción 
directa pueden transformar el medio y las relaciones sociales, generando comportamientos más sostenibles. Para ello se han perseguido los siguientes objetivos específicos que se plasman en las distintas fases de la investigación:

- Conocer e identificar modelos o prototipos contextuales en la vida infantil a partir de las dimensiones relacionadas con su contexto personal y familiar, el lugar de residencia y el modo de relacionarse con el medio urbano.

- Conocer la percepción que tienen los niños sobre los entornos urbanos de sus escuelas teniendo en cuenta diversas perspectivas.

- Conocer si existen diferencias en la percepción que tienen los niños atendiendo al modelo contextual en el que se inscribe.

- Transparentar la información objetiva obtenida para que pueda ser interpretada por la administración, agentes sociales y especialmente familias y niños, posibilitando el empoderamiento de estos últimos.

Para describir más adecuadamente la metodología llevada cabo nos apoyaremos en las cuatro partes que componen los Sistemas de Apoyo a la Decisión (Silver, 2008): (i) información y funciones de procesado, (ii) conjuntos de datos, (iii) modelos, y (iv) representaciones visuales.

(i) Información y funciones de procesado:

La información se ha obtenido a través de un muestreo estratificado, mediante la cumplimentación de formularios en papel por los alumnos de cuarto o quinto curso de Primaria de cada uno de los 21 centros educativos en los que fue posible llevar a cabo el estudio de los 33 Colegios Públicos de Granada. Se eligieron estos cursos porque, según los planes educativos, en ellos es cuando el alumnado ya ha recibido contenidos relacionados con la ciudad y la seguridad vial. La muestra fue de 459 niños de entre 10 y 11 años, edad en la que los niños comienzan a tener cierta autonomía en sus desplazamientos, incrementándose el número de padres que deciden dejar ir solos a sus hijos al colegio. A tal efecto se creó un formulario específico para la investigación, que se adaptó para la mejor comprensión de cada una de las cuestiones por parte de los niños. El cuestionario en su conjunto presentaba: a) 53 ítems; b) un plano del ámbito del colegio donde el niño debía representar algunas variables; c) un espacio para que el niño dibujara cómo le gustaría que fuera el recorrido desde su casa hasta el colegio; y d) otro espacio para que aportara una frase que describiera el camino. Se usaron 14 de las cuestiones para la cualificación de los aspectos contextuales y de entorno del niño, y otras 14 para las cuestiones perceptivas del recorrido hasta el colegio y sobre el entorno próximo al mismo. Se llevó a cabo un único instrumento, usándose el mismo formulario para la obtención de la información en las cuatro dimensiones descritas. En la Tabla 1 se puede observar una síntesis de los resultados de los cuestionarios.

En la sección del formulario destinada a la información contextual se abordaron tres dimensiones: (i) la dimensión personal y familiar (edad, género, centro educativo y persona/s con los que vive); (ii) la dimensión residencial (tipo de vivienda o alojamiento, si dispone de espacios como patios, jardín zonas para el juego deporte, etc.); y por último (iii) una dimensión consistente en su relación con el medio urbano, vinculada a la movilidad y al uso del tiempo extraescolar (tipos de vehículo usado en los desplazamientos de ida y vuelta, si va acompañado, actividades programadas o no fuera del horario escolar, etc.). Todas estas variables son de tipo categóricas (excepto la edad) por lo que, para su adecuado uso posterior, tuvieron que ser pre-procesadas y convertidas en variables dicotómicas o dummy. 
Tabla 1. Estadísticos de las dimensiones personal y familiar, residencial, relación con el medio, y perceptiva sobre el medio urbano del colegio de la muestra completa. Fuente: elaboración propia.

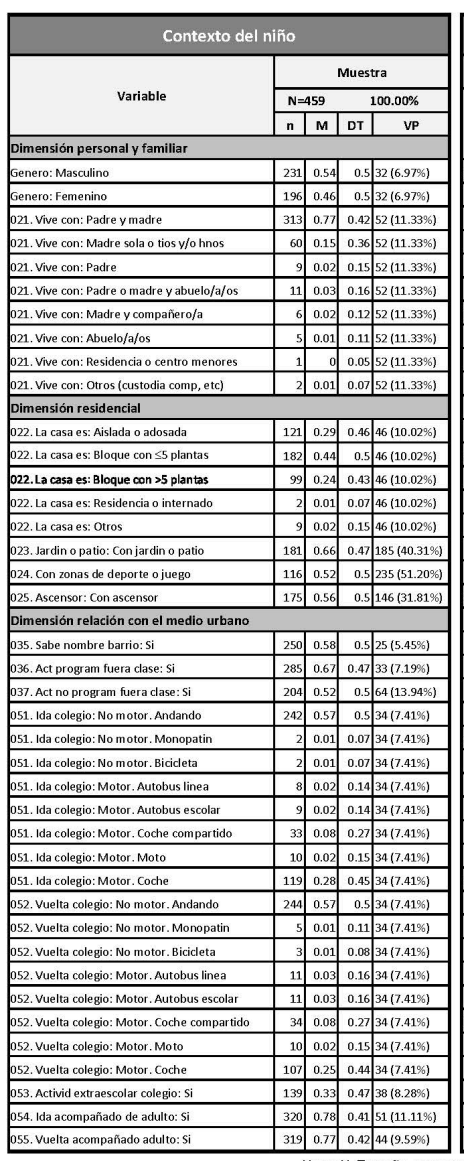

\begin{tabular}{|c|c|c|c|c|c|c|c|c|c|}
\hline \multicolumn{10}{|c|}{ Percepción del niño } \\
\hline \multicolumn{10}{|c|}{ Dimensión perceptiva del medio urbano del colegio } \\
\hline \multirow{3}{*}{ Variable } & \multicolumn{4}{|c|}{ Muestra } & \multirow{3}{*}{ Variable } & \multicolumn{4}{|c|}{ Muestra } \\
\hline & \multicolumn{2}{|c|}{$\mathrm{N}=459$} & \multicolumn{2}{|c|}{$100.00 \%$} & & \multicolumn{2}{|c|}{$\mathrm{N}=459$} & \multicolumn{2}{|c|}{$100.00 \%$} \\
\hline & $\mathrm{n}$ & $\mathrm{M}$ & DT & $\mathrm{VP}$ & & $\mathrm{n}$ & $\mathrm{M}$ & DT & $\mathrm{vP}$ \\
\hline 061. Disfruta con gente en recorrido & 418 & & \multirow{6}{*}{1.23} & \multirow{6}{*}{$\begin{array}{c}41 \\
(8.93 \%)\end{array}$} & 074i. (No) obstaculos desplazamientos & 417 & \multirow{6}{*}{2.09} & \multirow{6}{*}{\begin{tabular}{|l|}
1.2 \\
\end{tabular}} & \multirow{6}{*}{$\begin{array}{c}42 \\
(9.15 \%)\end{array}$} \\
\hline Valoración 5 & 103 & & & & Valoración 5 & 187 & & & \\
\hline Valoración 4 & 87 & & & & Valoración 4 & 83 & & & \\
\hline Valoración 3 & 158 & & & & Valoración 3 & 83 & & & \\
\hline Valoración 2 & 25 & & & & Valoración 2 & 40 & & & \\
\hline Valoración 1 & 45 & & & & Valoración 1 & 19 & & & \\
\hline 062. Contento entorno recorrido al colegio & \multirow{6}{*}{\multicolumn{2}{|c|}{ 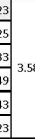 }} & \multirow{6}{*}{1.17} & \multirow{6}{*}{$\begin{array}{c}36 \\
(7.84 \%)\end{array}$} & 075. Contento con cantidad parques & 418 & & \multirow{6}{*}{1.38} & \multirow{6}{*}{$\begin{array}{c}41 \\
(8.93 \%)\end{array}$} \\
\hline Valoración 5 & & & & & Valoración 5 & 114 & & & \\
\hline Valoracion 4 & & & & & Valoración 4 & 88 & & & \\
\hline Valoración 3 & & & & & Valoración 3 & 98 & & & \\
\hline Valoración 2 & & & & & Valoración 2 & 59 & & & \\
\hline Valoración 1 & & & & & Valoración 1 & 59 & & & \\
\hline 063. Autonomia entorno colegio & 421 & & & & 076. Sensacion de entorno bonito & 412 & & & \\
\hline Valoración 5 & 81 & & & & Valoración 5 & 118 & & & \\
\hline Valoración 4 & 92 & & I & 38 & Valoración 4 & 95 & & 119 & 47 \\
\hline Valoración 3 & 157 & & ${ }^{1.18}$ & $(8.28 \%)$ & Valoración 3 & 131 & & & $(10.24 \%)$ \\
\hline Valoración 2 & 53 & & & & Valoración 2 & 42 & & & \\
\hline Valoracion 1 & 38 & & & & Valoración 1 & 26 & & & \\
\hline 064. Contento con autonomia & 423 & & & & 077. Sensacion entorno con historia & 414 & & & \\
\hline Valoración 5 & 168 & & & & Valoración 5 & 63 & & & \\
\hline Valoración 4 & 93 & 387 & 10 & 36 & Valoración 4 & 61 & & 137 & 45 \\
\hline Valoración 3 & 119 & & & $(7.84 \%)$ & Valoración 3 & 100 & & & $(9.80 \%)$ \\
\hline Valoración 2 & 23 & & & & Valoración 2 & 91 & & & \\
\hline Valoracion 1 & 20 & & & & Valoración 1 & 99 & & & \\
\hline 071. Contento con limpieza calles & 424 & & & & 078. Tiendas comprar lo que gusta & 412 & & & \\
\hline \begin{tabular}{|l|} 
Valoración 5 \\
\end{tabular} & 81 & & & & Valoración 5 & 111 & & & \\
\hline Valoración 4 & 71 & & 1 & 35 & Valoración 4 & 92 & & 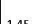 & 47 \\
\hline Valoración 3 & 143 & & 1.23 & $(7.63 \%)$ & Valoración 3 & 78 & & 1.45 & $(10.24 \%)$ \\
\hline Valoración 2 & 87 & & & & Valoración 2 & 56 & & & \\
\hline Valoración 1 & 42 & & & & Valoración 1 & 75 & & & \\
\hline 0721. (No) molesto cantidad de coches & 420 & & & & 079. Lugares que permiten juego & 417 & & & \\
\hline Valoración 5 & 107 & & & & Valoración 5 & 98 & & & \\
\hline Valoracion 4 & 98 & 年 & 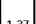 & 39 & Valoración 4 & 85 & & 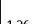 & 42 \\
\hline Valoración 3 & 92 & & 1.37 & $(8.50 \%)$ & Valoración 3 & 120 & & 1.26 & $(9,15 \%)$ \\
\hline Valoración 2 & 65 & & & & Valoración 2 & 78 & & & \\
\hline Valoracion 1 & 58 & & & & Valoración 1 & 36 & & & \\
\hline 073i. Trafico (no) impide jugar o desplam. & 418 & & & & 0710. Suele hacer recados & 415 & & & \\
\hline Valoración 5 & 178 & & & & Valoración 5 & 37 & & & \\
\hline Valoración 4 & 75 & & 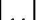 & 41 & Valoración 4 & 33 & & 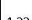 & 44 \\
\hline Valoración 3 & 67 & & 1.4 & (8.93\%) & Valoración 3 & 58 & 2.07 & 1.35 & (9.59\%) \\
\hline Valoración 2 & 53 & & & & Valoración 2 & 79 & & & \\
\hline Valoracion 1 & 45 & & & & Valoración 1 & 200 & & & \\
\hline
\end{tabular}

Nota: $\mathrm{N}=$ Tamaño muestra; $\mathrm{n}=$ Tamaño subconjunto; $\mathrm{M}=$ Media; $\mathrm{DT}=$ Desviación Típica; $\mathrm{VP}=$ Valores Perdidos.

La información perceptiva obtenida versa sobre los recorridos de la casa al colegio y sobre el entorno urbano inmediato al colegio, preguntando sobre el grado de satisfacción (¿Estás contento con...?, ¿Te molesta...?, ¿Tienes sensación....?, etc.): a) con la gente que se encuentra en los recorridos; b) con el entorno del recorrido; c) sobre la autonomía; d) sobre la limpieza; e) la cantidad de coches; f) sobre si el tráfico impide jugar o desplazarte; g) si hay obstáculos; h) sobre la cantidad de parques y lugares de juego; i) si el colegio está en un entorno bonito o con historia; j) si existen tiendas donde comprar las cosas que le gustan; y k) si suele hacer recados. Todas estas variables tienen una construcción en escala tipo Likert de cinco opciones de respuesta $(\mathrm{MP}=$ muy poco, $\mathrm{P}=$ poco, $\mathrm{N}=$ normal, $\mathrm{B}=$ bastante $\mathrm{y} \mathrm{M}=$ mucho).

(ii) Conjuntos de datos:

En el proyecto, inicialmente se opera con dos bases de datos conectadas: una contextual, correspondiente a las tres primeras dimensiones infantiles, y otra sobre 
percepción infantil (cuarta dimensión), con las que se operará tanto por separado como conjuntamente.

(iii) Modelos:

Para la creación del modelo de generación de conocimiento en base a la información obtenida sobre la realidad contextual de los niños, se usó la metodología llamada Mapa Auto-organizado (Self-Organizing Map - SOM-). Se trata de una técnica que tiene su origen en las Ciencias de la Inteligencia Artificial y Minería de Datos. Tienen la capacidad de representar la información mediante unas cartografías que permiten evaluar la organización de la realidad estudiada en patrones de correlación, similitud e influencia. Esta técnica está emparentada con otras técnicas de descubrimiento de patrones, como por ejemplo el Análisis Clúster tipo K-medias, alcanzando la red neuronal generalmente mejores resultados (Baçao et al, 2005), con la peculiaridad adicional de permitir mostrar las relaciones topológicas y de similitud entre los sujetos estudiados.

Los SOM se basan en una red neuronal artificial con aprendizaje competitivo no supervisado. Por ello no es necesaria la participación del usuario en la fase de entrenamiento de la red neuronal. Ésta se obtiene a partir de la entrada de una serie de atributos de los objetos de estudio (capa de entrada), una serie de patrones y la organización de los objetos de estudio (capa de salida o competición) formalizados en el Mapa Auto-organizado o SOM. Se obtiene así una cartografía no geográfica en la que cada instancia queda representada en un lugar único atendiendo a sus propiedades o atributos, mostrando alrededor las instancias que presentan mayor relación o conexión en sus propios atributos. Se trata de una representación topológica y relacional. Las metodologías SOM presentan una gran robustez en cuanto que son capaces de ser relativamente insensibles a los valores perdidos (VP).

Tras la generación de la cartografía SOM, se pueden representar agrupamientos de los sujetos mediante un análisis adicional llamado Ward-clúster, obteniendo la generación de perfiles o prototipos al modelar patrones y tendencias en la información (Weiss, 1998). Queda a elección del investigador el número de tales perfiles resultantes, ajustándose en nuestro caso a un número tal mediante el que sea posible interpretar los resultados obtenidos.

Una vez obtenido el conjunto de Perfiles mediante la técnica SOM, se procederá a la validación del modelo mediante la verificación estadística de la adecuación del mismo. Debido a que la fuente de conocimiento es fundamentalmente categórica (convertido en dicotómico, presencia-ausencia), se opta por realizar la prueba no paramétrica Chi-cuadrado, con la preceptiva corrección de Yates, en la que se contrasta si los resultados alcanzados para cada variable de estudio son independientes de la pertenencia o no a los distintos perfiles determinados.

Cuando quede verificada la adecuación del modelo SOM y de los Perfiles obtenidos, con la intención de alcanzar el segundo y tercer objetivo específico se procederá igualmente a evaluar si existe independencia o no entre las respuestas sobre la dimensión perceptiva y la pertenencia al Perfil.

Finalmente, siguiendo las recomendaciones de la American Statistical Association (Wasserstein y Lazar, 2016) se obtiene, para cada par de variable y perfil, el tamaño del efecto, entendido éste como la razón entre la probabilidad de que el evento ocurra y la probabilidad de que no ocurra. El índice "Odds Ratio" (OR) ha sido usado de forma común como índice del tamaño o "magnitud del efecto", siendo 
apropiado en resultados dicotómicos (Coe y Merino, 2003), como los de nuestro caso de estudio.

iv) Representaciones visuales:

Para el DSS y, en consecuencia, facilitar el empoderamiento ciudadano (cuarto objetivo específico), se plantea el uso de las cartografías SOM para la representación de la información útil y como método de síntesis y reducción de dimensiones de información multivariante disponible. Estas cartografías, combinadas con representaciones tipo Mapa de calor (Heatmap), acercan la información a los usuarios sin apenas instrucciones sobre su uso, permitiendo su evaluación e interpretación crítica $\mathrm{y}$, consecuentemente, el empoderamiento para la toma de decisiones.

\section{Análisis y resultados}

Siguiendo la metodología del apartado anterior se realizan en primer lugar las tareas propias de la sección (i) información y funciones de procesado y (ii) conjuntos de datos, obteniéndose dos bases de datos independientes. Un primer análisis descriptivo de las variables contextuales de la muestra indica una ligera mayor presencia en el estudio de niños que de niñas, con alto predominio de niños/as que viven con sus padres (68.19\%). No son desdeñables los hogares con la figura materna como principal adulto en casa (aprox. 13\%). Un 26.36\% viven en viviendas unifamiliares aisladas o adosadas, frente al $39.65 \%$ de aquellos que viven en un bloque de $\leq 5$ plantas, o el $21.56 \%$ que viven en un bloque de más de 5 plantas. Algo más de la mitad de los niños van y vienen al colegio a pie, frente al casi un cuarto de niño/as que se desplazan en coche. Por otro lado, un $69.49 \%$ de los niños van o vuelven acompañados por un adulto.

La fase (iii) modelo mediante el análisis SOM, se lleva a cabo usando 40 variables (muchas de ellas convertidas a dicotómicas a partir de las 28 categóricas iniciales) de las tres primeras dimensiones (contextuales): personal y familiar, residencial y de relación con el medio. Se realiza el agrupamiento en perfiles, llevándose a cabo para cada una de dichas variables, un contraste de independencia en cada uno de los 18 perfiles considerados (con un total de 720 contrastes de hipótesis). Asimismo, se ha calculado el Odds Ratio (OR) como medida del tamaño del efecto. Tales resultados se sintetizan en el gráfico Mapa de calor de la Figura 1. De este modo se consigue integrar y evaluar cómo las variables contextuales conforman unos perfiles. A su vez se evidencia la conexión de tales perfiles con la percepción que tienen los niños de la ciudad. Se debe tener en cuenta que la dimensión perceptiva nunca participó en la red neuronal para el cálculo del modelo de perfiles.

Para que la investigación sea más transparente y permita una mayor comprensión, es necesario el uso de (iv) representaciones visuales que aproximen de un modo sencillo la interpretación a cualquier usuario interesado no experto. Para ello se pretende usar la Figura 1, en la que se ha procesado gráficamente a modo de Mapa de calor: por un lado los OR con efecto positivo en tonos verdes; y por otro lado los OR con efecto negativo en tonos rojos. Adicionalmente los Efectos se presentan enmarcados mediante recuadro. Al ser significativos estadísticamente los contrastes de hipótesis, pueden ser tenidos en consideración para la toma de decisiones.

Mostramos a continuación algunos resultados obtenidos al analizar los perfiles: 
- Perfil 1: 17\% de la muestra en estudio. Mayor presencia de niñas en este perfil que viven mayoritariamente con el padre y la madre en casas de más de 5 plantas. Presentan una tendencia elevada a ir y venir al colegio andando y acompañados por un adulto. Los niños de este perfil tienen una ligera tendencia a realizar una valoración perceptiva más positiva que la del resto de los niños pertenecientes a otros perfiles. Esto se puede observar al comprobar que cierta parte de las valoraciones bajas (1 ó 2) tienen a bajar en número de frecuencias y de tamaño del efecto; y las más altas (4 ó 5) a subir ligeramente su frecuencia o tamaño del efecto. En este grupo apenas destacan valoraciones perceptivas que alcancen la significación estadística.

- Perfil 2: 20.88\% del total. En gran medida reside en vivienda aislada o adosada, desplazándose hasta el colegio en coche durante la ida y la vuelta. Destaca este perfil por la baja valoración perceptiva en numerosas cuestiones, como por ejemplo en relación a: los obstáculos, cantidad de parques, sensación de entorno bonito, sensación entorno con historia, tiendas donde comprar lo que le gusta o lugares que permiten el juego. En todas ellas, al menos un nivel de valoración ha presentado significación estadística.

- Perfil 3: 15.69\% sobre la totalidad. Elevado número de niños que viven con la madre sola o con tíos y/o hermanos en bloque de más de 5 plantas y desplazándose a pie mayoritariamente. Se observa una percepción mayoritariamente positiva, concretamente con unas frecuencias positivas mayores, y estadísticamente significativas en relación a: la autonomía, sobre los obstáculos, tiendas, lugares de juego o recados.

- Perfil 4: 12.85\% del total. Comparativamente elevado número de niños que viven con la madre sola, o con tíos y/o hermanos, o con abuelos, que se suelen alojar en viviendas de menos de 5 plantas, y suelen ir y volver al colegio andando o en algunos casos en monopatín. Suelen realizar actividades extraescolares. Ciertas valoraciones perceptivas son inferiores y estadísticamente significativas, como por ejemplo en relación a: la autonomía, al disfrute con la gente en el recorrido, baja sensación de entorno con historia, no siendo habitual la realización de recados. En otras variables, como por ejemplo: la molestia sobre la cantidad de coches, y si tiene percepción de que el tráfico le impide jugar, son superiores, aunque en este caso sin significación estadística.

- Perfil 5: 10.68\% del total. Con frecuencia de género masculino con mayor probabilidad de vivir con el padre y madre, que suele vivir en casa aislada, conoce el nombre del barrio donde vive, realiza actividades fuera de clase, y se desplaza andando ida y vuelta no acompañado por un adulto. Curiosamente no se muestra contento con su alta autonomía, reduciéndose drásticamente las valoraciones altas sobre los lugares del juego. En cambio, valoran de forma elevada la sensación histórica, reconociendo con una mayor probabilidad que suelen hacer recados. A otro nivel también y sin significación estadística, valoran positivamente los parques, los obstáculos, y no manifiestan especiales molestias por el tráfico.

- Perfil 6: 7.19\% del total. Suele residir en una vivienda aislada o adosada, y una mayoría se desplaza en coche compartido. A nivel perceptivo, de forma estadísticamente significativa: disfruta menos de la gente en el recorrido, tiene menos sensación de entorno bonito, menor valoración sobre las tiendas. 
En menor medida (sin significación) podemos destacar la valoración negativa: sobre los lugares de juego, sensación de entorno con historia, cantidad de parque, sobre limpieza de calles o sobre su valoración sobre el entorno.

Figura 1. Mapa de calor de la significación y Tamaño del efecto (Odds Ratio) de la pertenencia a un Perfil y la dimensión personal, residencial, de relación con el medio urbano y perceptiva. Fuente: elaboración propia.

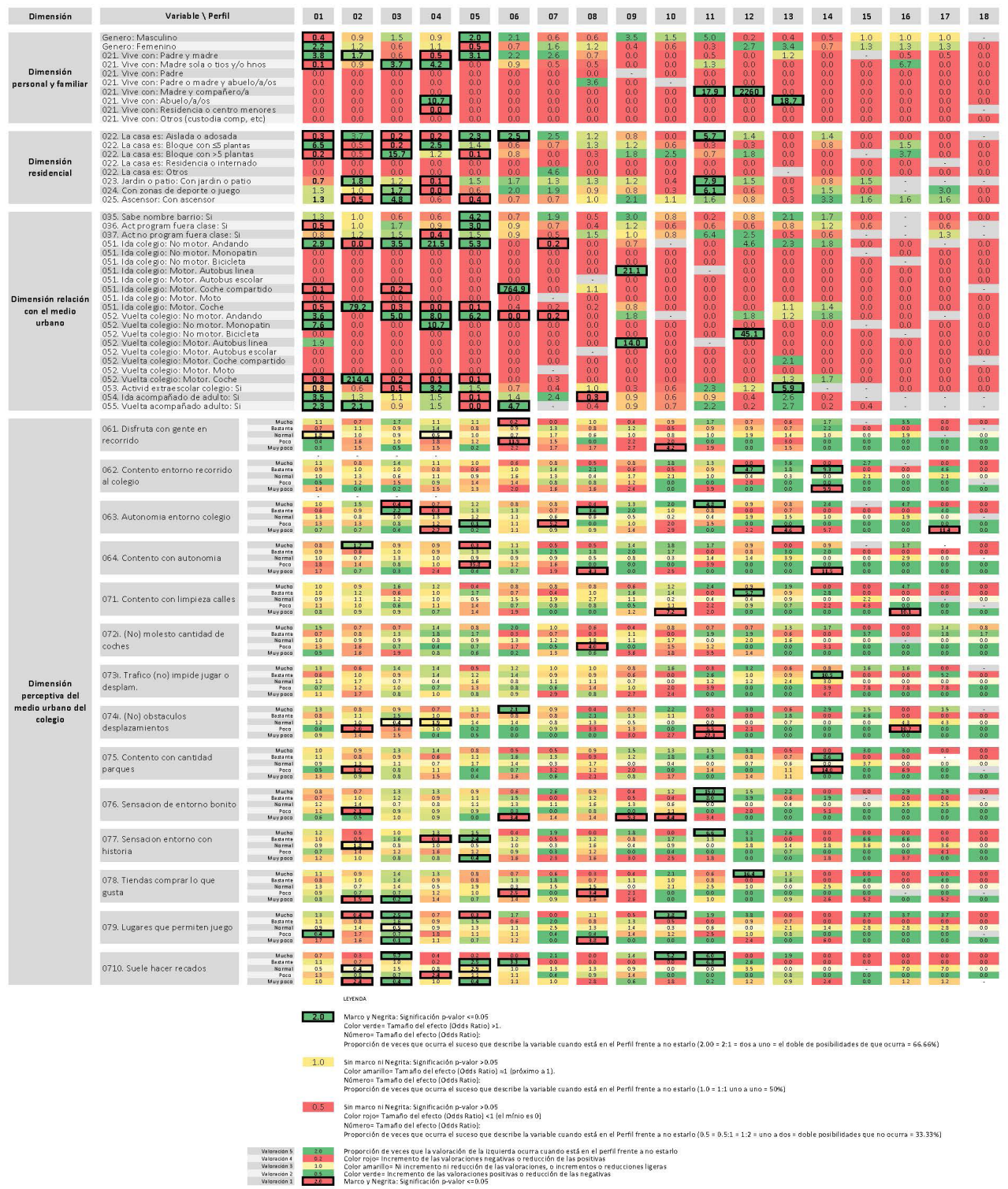

En otros perfiles minoritarios emergen especialmente otros modos de desplazamiento. Así, en el Perfil 7: utilizan la moto y valoran negativamente la autonomía. 
En el Perfil 8 utilizan el autobús escolar, y valoran de forma positiva la cantidad de coches, los obstáculos de la ciudad, cantidad de parques, la historia de la ciudad y lugares para el juego, presentando una alta autonomía aunque valorada de forma negativa. Por su lado el Perfil 9 suele ser: niño que se mueve en autobús de línea, valorando positivamente su autonomía, no disfrutando con la gente, ni con el entorno del recorrido, molesto con el tráfico, no valorando que sea un entorno bonito, ni apreciando las tiendas.

\section{Discusión y conclusiones}

Las ciencias de la información tienen la capacidad de mejorar los procesos de participación, proporcionando nuevos niveles de compromiso e involucrando al ciudadano en el diseño de los procesos y en el contenido de la formulación de las políticas (Caddy y Vergez, 2001). Este trabajo se enmarca en tales consideraciones, con la finalidad de generar conocimiento útil y relevante basado en la perspectiva infantil sobre entornos urbanos escolares. Este conocimiento complejo, que relaciona lo objetivo de la ciudad con lo perceptivo de sus habitantes en una situación y contexto concreto, al ser mostrado de un modo conciso y asequible a todo el mundo, es muy útil y facilita el empoderamiento ciudadano en la comprensión para la toma de decisiones.

La metodología elegida en el proyecto, la Investigación Participación-Acción, es idónea para implicar a los agentes involucrados por su vertiente eminentemente activa y colaborativa, siendo singularmente relevante por: su capacidad de crear comunidades críticas, de iniciarse en pequeños grupos de colaboradores expandiéndose paulatinamente a grupos mayores, generando progresivamente cambios más amplios y avanzando hacia problemas cada vez de mayor envergadura (Kemmis y McTaggart, 1988). Se convierte así en todo un aliado para los procesos en los que se persigue el empoderamiento ciudadano para las decisiones, colaborando en la construcción de una sociedad más crítica.

En el desarrollo de la investigación se han detectado una serie de dificultades y limitaciones que son propios y frecuentes en los DSS (Silver, 1990), debiendo ser tenidas en cuenta para su gestión anticipada: (i) limitaciones funcionales por la necesidad de un equipo de técnicos; (ii) limitaciones de los modelos en futuras situaciones más complejas; (iii) limitaciones de los parámetros con la incorporación de nuevas categorías; y (iii) limitaciones de las representaciones gráficas que puedan dificultar la comprensión ciudadana.

Como resultado de la investigación, en línea con sus objetivos, se han determinado y caracterizado tres patrones básicos de comportamiento que vinculan las dimensiones contextuales (personal y familiar, residencial y de relación con el medio urbano), con la dimensión de la percepción que tiene el niño del entorno urbano alrededor de su colegio:

- Patrón A: Existe predominio de una percepción del niño significativamente más positiva de la realidad del entorno escolar. Coincide con las organizaciones familiares más frecuentes y en las que el niño se desplaza fundamentalmente a pie. Con este patrón podemos encontrar los Perfiles 1, 3, 5, 12 , $13, \mathrm{y} 15$. 
- Patrón B: Valoración perceptiva intermedia, con determinadas variables valoradas por encima de la media y viceversa. Presentan cierta singularidad en su estructura familiar, por su relativamente baja frecuencia. Entre estos perfiles podemos encontrar: a) el Perfil 4, que destaca el que el niño vive sólo con su madre; b) el Perfil 10, en el que es frecuente que el niño viva con el padre, con la madre, o con abuelos; c) el Perfil 11, residiendo habitualmente con su madre y compañeros o con sus abuelos; y d) Perfiles minoritarios, como por ejemplo el Perfil 14, con niños que viven en centros de menores; el Perfil 16, con niños que suelen desplazarse en bicicleta; o el Perfil 17, de niños con residencia en un internado.

- Patrón C: Es significativa una inferior valoración perceptiva de la realidad en numerosas variables. En este patrón el niño suele desplazarse con medios motorizados, siendo: frecuentemente en coche en el Perfil 2, en coche compartido en el Perfil 6, en moto en el Perfil 7, en autobús escolar en el Perfil 8 y en autobús de línea en el Perfil 9.

Los resultados obtenidos se enmarcan en el interés actual por la caminabilidad (o walkability). Viene a suponer una renovada atención de la planificación, el diseño urbano y la toma de decisiones por la dimensión humana, sumergiéndose en el ámbito de la sostenibilidad (Mack, 2012). Cuando algo tan cotidiano, como puede ser el tránsito de los niños y adultos al colegio, se realiza caminando cómodamente, se incentiva un aumento del capital social y de la calidad de vida, reduciendo el aislamiento y mejorando las relaciones del vecindario. Poniéndole límites al coche se conseguirá fomentar el tránsito peatonal sobre el rodado, evitar la congestión y reducir la contaminación. Se trata simultáneamente de fomentar el aprendizaje a través de estos itinerarios, que deberán evitar ser discontinuos o cortos para evitar un aprendizaje superficial y parcial (Trilla, 1993). Hay un entusiasmo creciente por este tipo de iniciativas pero faltan mediadores y profesionales que puedan educar en esta línea, así como indicadores de evaluación para medir la repercusión de este tipo de educación en la sociedad y en el medio (Uttke, 2012; Trilla, 1993).

Por estas razones, el tipo de trabajo que se presenta se cree relevante, en el sentido de que ha permitido una construcción colectiva de conocimiento a través de un proceso colaborativo y autopedagógico. La información generada es necesaria para continuar el proceso con debates y talleres de modo más informado y concienciado por técnicos y ciudadanía no experta, e incluso por los propios niños, hacia la construcción de los entornos urbanos que se consensuen como más adecuados.

Mediante la presente investigación se consigue demostrar, para el caso específico de estudio, que el contexto en el que se desenvuelve el niño en sus dimensiones familiar, residencial y de relación con el medio urbano, afecta de forma significativa a la percepción que tiene del propio medio urbano que le rodea, lo que podría ser motor o inhibidor de determinados comportamientos sociales y de relación, condicionando las capacidades educativas y de empoderamiento de la ciudad. Se ha pretendido profundizar en la "socio-práxis" (CIMAS, 2015), es decir, intentar alcanzar y desbordar las ya clásicas metodologías cuantitativas y cualitativas, e incorporar la creatividad colectiva como un factor de gran importancia para el conocimiento social (Shukaitis y Graeber, 2007).

Desde el máximo número de ámbitos posibles, —educación formal e informal, planificación, etc. - se deben hacer todos los esfuerzos para devolver al niño el 
espacio que nunca debió perder: un espacio físico en la ciudad, pero también mental. Incentivando que expresen sus sentimientos, sus quejas, necesidades, anhelos y deseos. Evitando quebrantar su derecho a expresar su opinión y a que ésta se tenga en cuenta en los asuntos que le afectan (Tonucci, 1996), hasta llegar a convertirlo en actor, participando en la realidad con sus propias decisiones, incentivando cambios en las costumbres familiares, construyendo el desenlace de la ciudad como soporte de la ciudadanía crítica y de la justicia social.

\section{Referencias}

Abarca-Álvarez, F. J. (2011). Identificación de patrones para la ordenación urbanística mediante redes neuronales: Hacia la Ordenanza-Red. Serie Geográfica, 17, 45-60. Disponible en http://hdl.handle.net/10481/18495.

Abarca-Álvarez, F. J., Osuna-Pérez, F. (2013). Cartografías semánticas mediante redes neuronales: los mapas auto-organizados (SOM) como representación de patrones y campos. EGA: Revista de Expresión Gráfica Arquitectónica, 18 (22) 154-163. Disponible en https://doi.org/10.4995/ega.2013.1692.

Abarca-Álvarez, F. J., Campos-Sánchez, F. S. y Osuna-Pérez, F. (2015). Taxonomía de las inmigraciones turísticas de Andalucía basada en las cualidades de sus asentamientos urbanos. En I Congreso Internacional de migraciones contemporáneas, territorio y urbanismo: Actas del congreso internacional, Cartagena, 9 y 10 de julio de 2015. Cartagena: Universidad Politécnica de Cartagena, Servicio de Documentación, 123-137. Disponible en http://hdl.handle.net/10317/5112.

Abarca-Álvarez, F. J., Fernández Avidad, A. (2010). Generation of Downtown PlanningOrdinances using Self Organizing Maps. Design \& Decision Support Systems 2010 International Conference. Eindhoven: Tue Eindhoven.

Arampatzis, G., Kiranoudis, C. T., Scaloubacas, P. y Assimacopoulos, D. (2004). A GISbased decision support system for planning urban transportation policies. European Journal of Operational Research, 152 (2), 465-475.

Bação, F., Lobo, V. y Painho, M. (2005). Self-organizing maps as substitutes for k-means clustering. Computational Science-ICCS 2005, 3516, 476-483. Disponible en http://doi. org/10.1007/11428862_65.

Baierle, T. y Cayford, J.(2001). Democracy in Practice: Public Participation in Environmental Decisions. Washington DC, USA: Resources for the Future.

Bauman, Z. (2003). Modernidad Líquida. Argentina: Fondo de cultura económica.

Bray, J. y McLaughlin, D. (2005). Getting to Ground: Democratic Renewal in Canada. Crossing Boundaries Papers. Ottawa, USA: The Crossing Boundaries National Council.

Caddy, J. y Vergez C. (2001). Citizens as Partners: Information, Consultation and Public Participation in Policy-making. Organisation for Economic Co-operation and Development.

Cao, L. (2009). Introduction to Domain Driven Data Mining. En L. Cao, S. Y. Philip, C. Zhang, y H. Zhang, Eds. Data mining for business applications. New York, USA: Springer Science+Business Media, LLC. http://doi.org/10.1007/978-0-387-79420-4

Carlsson, S. A. y El Sawy, O. A. (2008). Decision Support in Turbulent and HighVelocity Environments. Chapter 37. En F. Burstein y W. Holsapple (Eds). Handbook on Decision Support Systems 2: Variations (pp. 3-17). Berlin Heidelberg, Alemania: Knowledge Management. Springer-Verlag. http://doi.org/10.1007/978-3-540-48713-5 
Castells, M. (1995). La ciudad informacional: tecnologías de la información, reestructuración económica y el proceso urbano-regional. Madrid: Alianza.

CIMAS Red, Alberich, T., Arnanz, L., Basagoiti, M., Belmonte, R., Bru, P., Espinar, C., García, N., Habegger, S., Heras, P., Hernández, D., Lorenzana, C., Martín, P., Montañés, M., Villasante, T. R. y Tenze, A. (2015). Metodologías Participativas: Sociopraxis para la creatividad social. Madrid: Dextra Editorial.

Coe, R. y Merino, C. (2003). Magnitud del efecto: Una guía para investigadores y usuarios. Revista de Psicología, 21(1), 147-177.

Crick, B. (2002). Democracy: A Very Short Introduction. Oxford, UK: Oxford University Press.

De Almeida, E. L., Davis, G., Zanoni, E. y de Souza Mendes, L. (2013). Knowledge Discovery: Data Mining by Self-organizing Maps. En J. Cordeiro y K. H. Krempels (Eds.): WEBIST 2012, LNBIP 140 (pp. 185-200). Berlin Heidelberg, Alemania: Springer-Verlag.

Diappi, L., Bolchim, P. y Buscema, M. (2004). Improved Understanding of Urban Sprawl Using Neural Networks. Dordrecht, Países Bajos: Springer.

Dryzek, J. S. (1990). Discursive Democracy. New York, USA: Cambridge University Press.

Feng, S. y Xu, L. D. (1999). Decision support for fuzzy comprehensive evaluation of urban development. Fuzzy Sets and Systems, 105(1), 1-12.

Freire, P. (1970). Pedagogia do Oprimido. Rio de Janeiro, Brasil: Paz e Terra.

Garnier, J. P. (2006). Contra los territorios del poder. Por un espacio público de debates y de combates. Barcelona, España: Virus.

Goyette, G. y Lessard-Hébert, M. (1988). La investigación-acción: Funciones, fundamentos e instrumentación. Barcelona, España: Laertes.

Habermas, J. (1994). Three Normative Models of Democracy. Constellations, 1(1),1-10.

Hagen, A. y Timmermans, H. J. P. (2009). A metric of compactness of urban change illustrated to 22 European countries. European Information Society: Taking Geoinformation Science One Stepfurther, 181-200.

Harvey, D. (2013). Rebel Cities: From the right to the city to the urban revolution. London, UK: Verso.

Hessel, S. (2011). ¡Indignaos!: Un alegato contra la indiferencia y a favor de la insurrección pacifica. Prologado en España por José Luis Sampedro. Barcelona, España: Destino.

Irwin, E. G. y Geoghegan, J. (2001). Theory, data, methods: Developing spatially explicit economic models of land use change. Agriculture Ecosystems and Environment, 85(1-3) 7-23.

Keen, P. G. (1987). Decision support systems: the next decade. Decision Support Systems, 3(3), 253-265.

Kemmis, S. y Mctaggart, R. (1988). Cómo planificar la investigación-acción. Barcelona, España: Laertes.

Kohonen, T. (1989). Self-organizing semantic maps. Biological cybernetics, 61(4) 241-254.

Kropp, J. (1998). A neural network approach to the analysis of city systems. Applied Geography, 18(1) 83-964.

Lefebvre, H. (1968). Le Droit à la ville. París, France: Anthropos.

Mack, E. A. (2012). Review of the book: Sustainability in America's Cities: Creating the Green Metropolis. Journal of Planning Literature, 27(4), 461-462.

Nel-Lo, O. (2015). La ciudad en movimiento. Madrid, España: Díaz \& Pons.

Oliverira de Vasconcelos, V. y Waldemez de Oliveira, M. (2010). Trayectorias de investigación acción: Concepciones, objetivos y planteamientos. Revista Iberoamericana de Educación, 53(5), 13. http://www.rieoei.org/deloslectores/3390Oliveira.pdf 
Openshaw, S. y Abrahart, R. J. (2000). Geocomputation. London and New York, UK, USA: Taylor and Francis.

Power, D. J., Sharda, R. y Burstein, F. (2002). Decision support systems. New Yersey, USA: John Wiley \& Sons, Ltd.

Riechmann, J. (2011). Frente al abismo. Papeles de relaciones ecosociales y cambio global, 115, 27-48.

Rifkin, J. (2014). La sociedad de coste marginal cero: El internet de las cosas, el procomún colaborativo y el eclipse del capitalismo. Barcelona, España: Espasa Libros, S. L. U.

Ríos, D., Kersten, G. E. y Grima, C. (2008). Towards Decision Support for Participatory Democracy. Chapter 66. En F. Burstein y W. Holsapple, C. (Eds.). Hand book on Decision Support Systems 2: Variations (pp. 652-685). Berlin Heidelberg, Alemania. Knowledge Management. Springer-Verlag. http://doi.org/10.1007/978-3-540-48713-5.

Sassen, S. (2013). Entrevista a Saskia Sassen: Urbanismo de código abierto y smart cities. Disponible en http://www.paisajetransversal.org/2013/02/entrevista-saskia-sassen-urbanismo-de.html.

Shukaitis, S y Graeber, D. Eds. (2007). Constituent Imagination. Militant organizations through participation. Amsterdam, Países Bajos: John Benjamins.

Silver, M. S. (1990). Decision Support Systems: Directed and Non-Directed Change. Inform Sys Res, 1 (1), 47-70.

Silver, M. S. (2008). On the Design Features of Decision Support Systems: The Role of System Restrictiveness and Decisional Guidance. Chapter 49. En F. Burstein y W. Holsapple, (Eds.). Hand book on Decision Support Systems 2: Variations (pp. 261-291). Berlin Heidelberg, Alemania: Knowledge Management. Springer-Verlag. http://doi. org/10.1007/978-3-540-48713-5

Sloterdijk, P. (2006). Esferas III. Espumas. Madrid, España: Siruela.

Spielman, S. E. y Thill, J. C. (2008). Social area analysis, data mining, and GIS. Computers. Environment and Urban Systems, 32 (2) 110-122.

Thiollent, M. (1981). Investigación-acción. Chasqui. Revista Latinoamericana de Comunicación, (1), 76-78. http://doi.org/http://dx.doi.org/10.16921/chasqui.v0i1.975. g1009.

Tonucci, F. (1996). La ciudad de los niños. Madrid, España: Grao.

Trilla, J. (1993). La educación fuera de la escuela. Ámbitos no formales y educación social. Barcelona, España: Ariel.

Uttke, A. (2012). Towards the future design and development of cities with built environment education: Experiencies of scale, methods, and outcomes. Procedia. Social and behavioral sciences, 45, 3-13.

Wasserstein, R. L., Lazar, N. A. (2016). The ASA's statementon p-values: context, process, and purpose. The American Statistician, 1305 (April), 00-00. http://doi.org/10.1080/000 31305.2016.1154108

Weiss, S. M. y Indurkhya, N. (1998). Predictive Data Mining: A Practical Guide. San Francisco, USA: Morgan Kaufmann.

White, R. y Engelen G. (1993). Cellular-automata and fractal urban form a cellular modeling approach to the evolution of urban land use patterns. Environment and Planning A, 25(8), 1175-1199.

Wu, F. (1998). An experiment on the generic polycentricity of urban growth in a cellular automaticcity. Environment and Planning B Planning and Design, 25(5), 731-752. 